\title{
Temperature oscillations of magnetization observed in nanofluid ferromagnetic graphite
}

\author{
S Sergeenkov ${ }^{1,3}$, N S Souza ${ }^{1}$, C Speglich ${ }^{1}$, V A G Rivera ${ }^{1}$, C A \\ Cardoso $^{1}$, H Pardo ${ }^{2}$, A W Mombrú ${ }^{2}$ and F M Araújo-Moreira ${ }^{1,4}$ \\ ${ }^{1}$ Materials and Devices Group, Department of Physics and Physical Engineering, \\ Universidade Federal de São Carlos, São Carlos, SP, 13565-905 Brazil \\ ${ }^{2}$ Crystallography, Solid State and Materials Laboratory (Cryssmat-Lab), \\ DEQUIFIM, Facultad de Química, Universidad de la República, P.O. Box 1157, CP \\ 11800, Montevideo, Uruguay \\ ${ }^{3}$ Corresponding author, E-mail: sergei@df.ufscar.br \\ ${ }^{4}$ Research Leader, E-mail: faraujo@df.ufscar.br
}

\begin{abstract}
We report on unusual magnetic properties observed in the nanofluid roomtemperature ferromagnetic graphite (with an average particle size of $l \simeq 10 \mathrm{~nm}$ ). More precisely, the measured magnetization exhibits a low-temperature anomaly (attributed to manifestation of finite size effects below the quantum temperature $T_{l} \propto \hbar^{2} / l^{2}$ ) as well as pronounced temperature oscillations above $T=50 \mathrm{~K}$ (attributed to manifestation of the hard-sphere type pair correlations between ferromagnetic particles in the nanofluid).
\end{abstract}

PACS numbers: $75.50 \mathrm{Dd}, 75.50 \mathrm{Mm}, 75.50 . \mathrm{Tt}, 81.05 . \mathrm{Uw}, 82.70 . \mathrm{Kj}$ 
Recently, quite a substantial progress has been made in developing suspended colloids of nano sized magnetic particles, including carbon, graphite and graphene based nanofluids and biocompatible ferrofluids (see, e.g.,[1-9] and further references therein). In particular, Parkansky et al [6] successfully separated magnetic carbon particles (including chains of nanospheres with diameters from 30 to $50 \mathrm{~nm}$, and nanorods with lengths from 50 to $250 \mathrm{~nm}$ and diameters from 20 to $30 \mathrm{~nm}$ ) in the obtained solutions by means of the bio-ferrography technique. At the same time, Widenkvist et al [9] suggested a new method to produce suspensions of graphene sheets (graphite flakes) by combining solution-based bromine intercalation and mild sonochemical exfoliation.

In this paper, we report on the magnetic properties of the nanofluid magnetic graphite (NFMG) obtained from the previously synthesized bulk organic magnetic graphite (MG) by stabilizing the aqueous ferrofluid suspension with an addition of active cationic surfactant. Two interesting phenomena have been observed in the temperature behavior of the magnetization: a low-temperature anomaly (attributed to the manifestation of quantum size effects due to an average particle size of the order of $l \simeq 10 \mathrm{~nm}$ ), and pronounced temperature oscillations above $T=50 \mathrm{~K}$ (attributed to manifestation of the hard-sphere type pair correlations between ferromagnetic particles in the nanofluid).

Recall [10-13] that the chemically modified magnetic graphite (MG) was produced by a vapor phase redox controlled reaction in closed nitrogen atmosphere with addition of copper oxide using synthetic graphite powder. The obtained in such a way modified graphite has a strong magnetic response even at room temperature (which manifests itself through a visible attraction by a commercial magnet). After obtaining the MG, we have prepared the nanofluid suspension (NFMG) by dissolving graphite in acetone, adding a Cetyltrimethylammonium bromide (CTAB) cationic surfactant, and bringing it to an ultra sonic edge. The structural characterization of NFMG was performed by transmission electron microscopy (TEM) using Philips CM-120 microscope. The analysis of TEM images (shown in Fig.1) reveals clusters (ranging from 100 to 300nm) with an average size of the ferromagnetic particle in the nanofluid of the order of $10 \mathrm{~nm}$ (more details regarding the structure and chemical route for synthesis of the discussed here nanofluid magnetic graphite will be presented elsewhere [14]).

To test the magnetic properties of NFMG samples, we performed the standard zero field cooled (ZFC) and field cooled (FC) measurements using a MPMS-5T SQUID magnetometer from Quantum Design. Fig 2 presents the temperature dependence of the normalized magnetization $M(T) / M\left(T_{p}\right)$ (taken under the applied magnetic field of $1 \mathrm{kOe})$ after subtraction of paramagnetic contributions $\left(T_{p}=0.16 T_{C}=48 \mathrm{~K}\right.$ is the temperature where $M(T)$ has a maximum with the absolute value of $M\left(T_{p}\right)=$ $0.1 \mathrm{emu} / \mathrm{g}$ ). Notice that there are two distinctive regions, below and above the peak temperature $T_{p}$. Namely, below $T_{p}$ there is a well-defined low-temperature minimum (around $T_{m}=0.05 T_{C}=15 K$ ), while for $T>T_{p}$ we have pronounced temperature oscillations. To verify that the observed peak originates from true quantum effects in ferromagnetic sample (rather than from superparamagnetic behavior due to the 


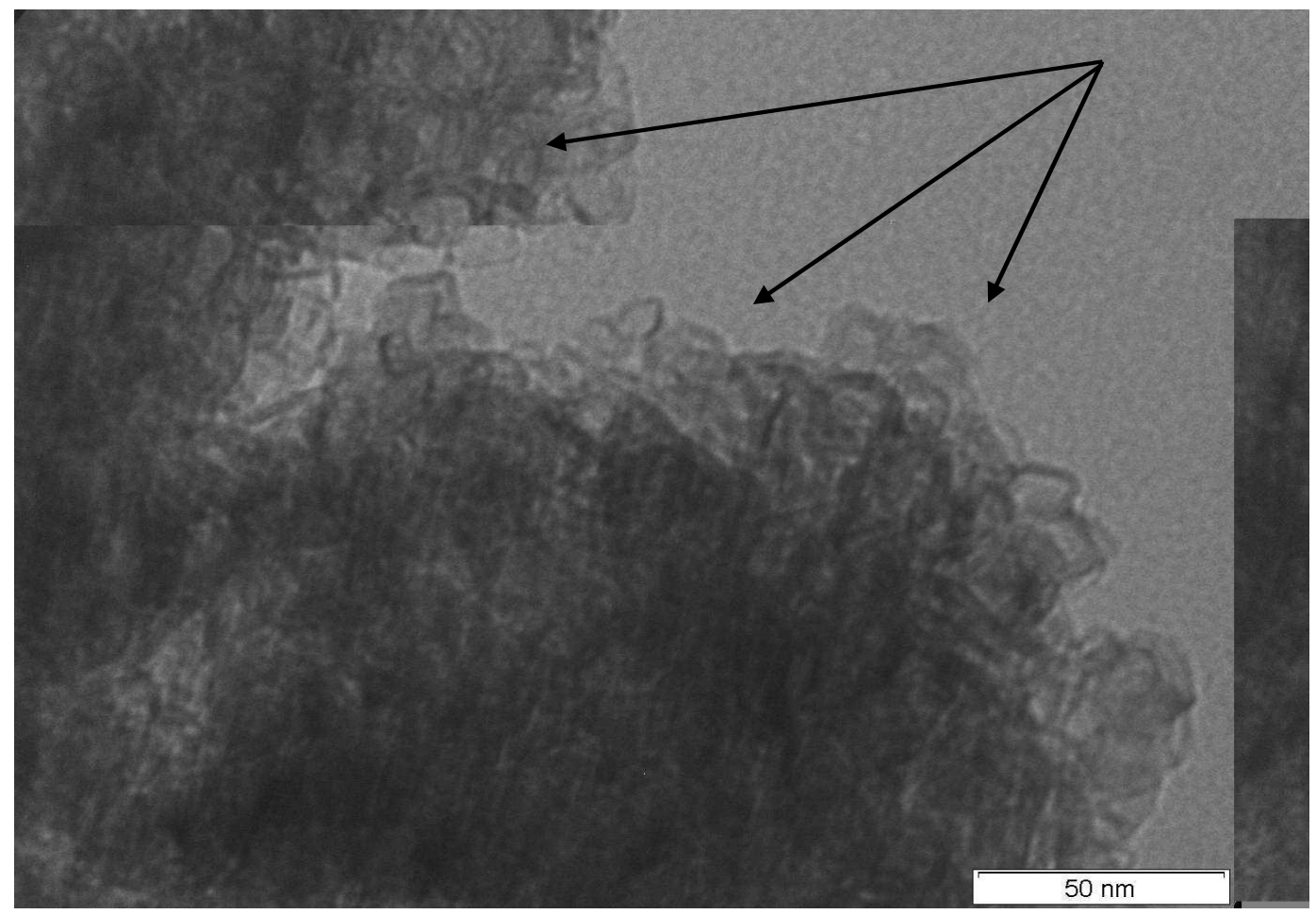

Figure 1. TEM image of NFMG sample.

thermal energy domination over anisotropy energy), we also measured the hysteretic $M-H$ curves for two characteristic temperatures, $T=5 K<T_{m}$ (in the region of fully fledged quantum effects) and $T=150 \mathrm{~K}=0.5 T_{C}$ (in the middle of the oscillations pattern). According to Fig, 3 , the low-temperature hysteresis is quite strong (with coercive magnetic field $H_{C}=3380$ ) and it does not disappear with increasing the temperature $\left(H_{C}=200 O e\right.$ for $\left.T=150 K\right)$. Thus, we can safely assume that the observed temperature features in our sample do originate from a true ferromagnetic behavior.

Turning to the analysis of the obtained results, let us begin with the lowtemperature region $\left(T<T_{p}\right)$ and discuss the origin of the observed minimum of magnetization near $T_{m}=0.05 T_{C}$. Recall that the finite temperature quantum effects manifest themselves for the size of the particle $l<\Lambda(T)$ (where $\Lambda(T)=\sqrt{2 \pi \hbar^{2} / m^{*} k_{B} T}$ is the thermal de Broglie wavelength) or, alternatively, for temperatures $T<T_{l}$ (where $T_{l}=2 \pi \hbar^{2} / m^{*} k_{B} l^{2}$ is the quantum temperature). Using $l \simeq 10 \mathrm{~nm}$ for an average size of the single particle in our samples (and assuming free electron mass for $m^{*}$ ), we get $T_{l}=0.15 T_{C}=45 \mathrm{~K}$ for the onset temperature below which the manifestation of quantum size effects is expected (notice that $T_{l}$ is very close to the peak temperature $T_{p}=0.16 T_{C}$ ). To fit the low-temperature experimental data, we assume the following normalized (to the peak temperature $T_{p}$ ) periodic dependence of the finite-size magnetization:

$$
\frac{M_{l}(T)}{M_{l}\left(T_{p}\right)}=\left[\frac{l}{\Lambda(T)}\right] \sin \left\{\left[\frac{M_{\infty}(T)}{M_{\infty}\left(T_{p}\right)}\right]\left[\frac{\Lambda(T)}{l}\right]\right\}
$$


Temperature oscillations of magnetization observed in nanofluid ferromagnetic graphite4

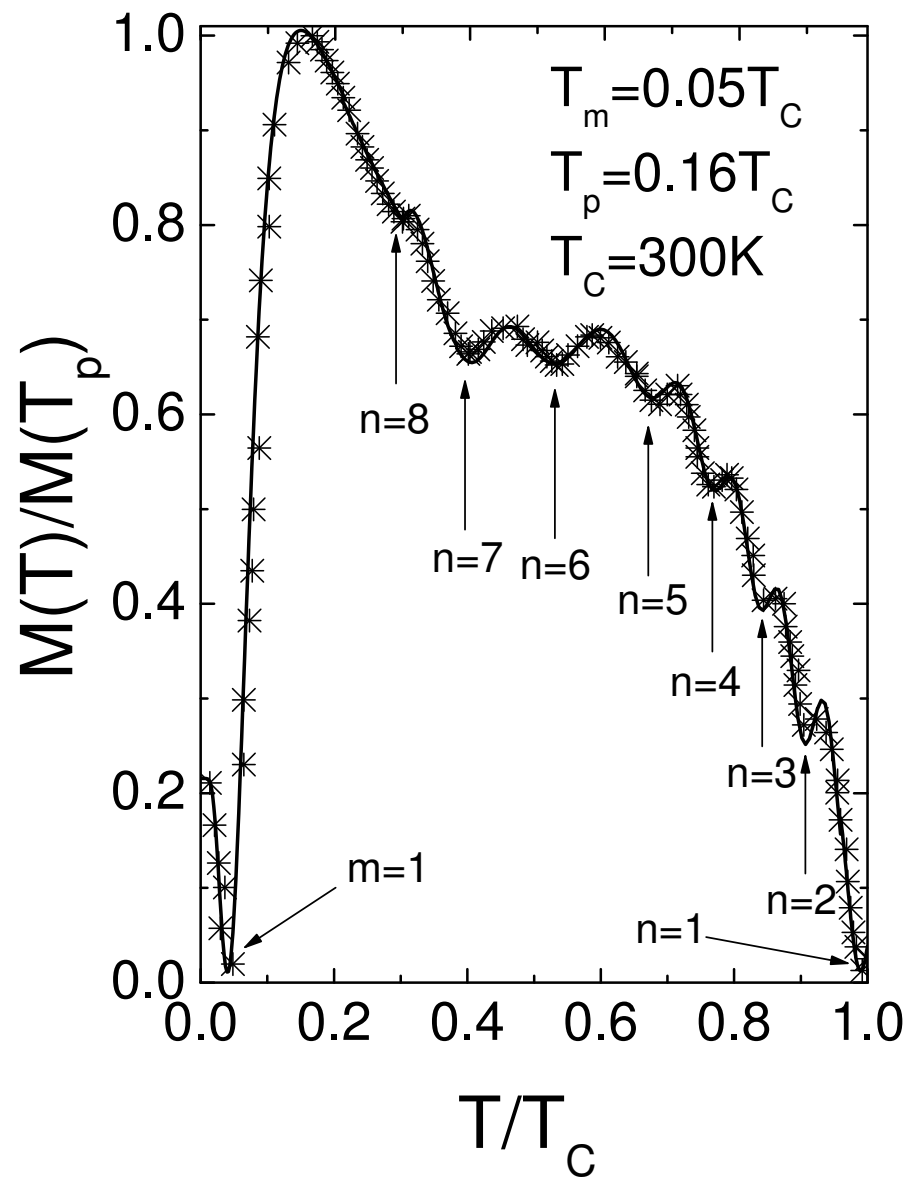

Figure 2. The temperature dependence of the normalized magnetization of NFMG (after subtracting the paramagnetic contribution). The solid line is the best fit according to Eqs.(1)-(3).

where $M_{\infty}(T)$ is the bulk magnetization of a single magnetic particle.

It can be easily verified that Eq.(1) reduces to $M_{\infty}(T)$ when the quantum effects become negligible. More precisely, $M_{\infty}(T) / M_{\infty}\left(T_{p}\right)=\lim _{l \gg \Lambda(T)}\left[M_{l}(T) / M_{l}\left(T_{p}\right)\right]$.

We were able to successfully fit the low-temperature data using the following explicit expression for the single particle bulk magnetization:

$$
M_{\infty}(T)=M_{s} \tanh \sqrt{\left(\frac{T_{C}}{T}\right)^{2}-1}
$$

which presents analytical (approximate) solution of the Curie-Weiss mean-field equation for spontaneous magnetization valid for all temperatures $[15,16]$. The solid line in Fig[2 presents the best fits for low-temperature region $\left(T \leq T_{p}\right)$ according to Eqs.(1) and (2) with $M_{s}=0.95 M\left(T_{p}\right), M\left(T_{p}\right)=0.1 \mathrm{emu} / g, T_{C}=300 \mathrm{~K}$ and $l=10 \mathrm{~nm}$.

Notice also that, for a given temperature, the above periodic function $M_{l}(T)$ has minima $m$ at $T=T_{m}$ where $T_{m}$ is the solution of the implicit equation, $M_{\infty}\left(T_{m}\right) \Lambda\left(T_{m}\right)=$ $\pi m M_{\infty}\left(T_{p}\right) l$ with $m=1,2, \ldots$ being the number of the oscillation minima. Using the Curie-Weiss expression for bulk magnetization $M_{\infty}(T)$ and previously defined thermal 
Temperature oscillations of magnetization observed in nanofluid ferromagnetic graphite5
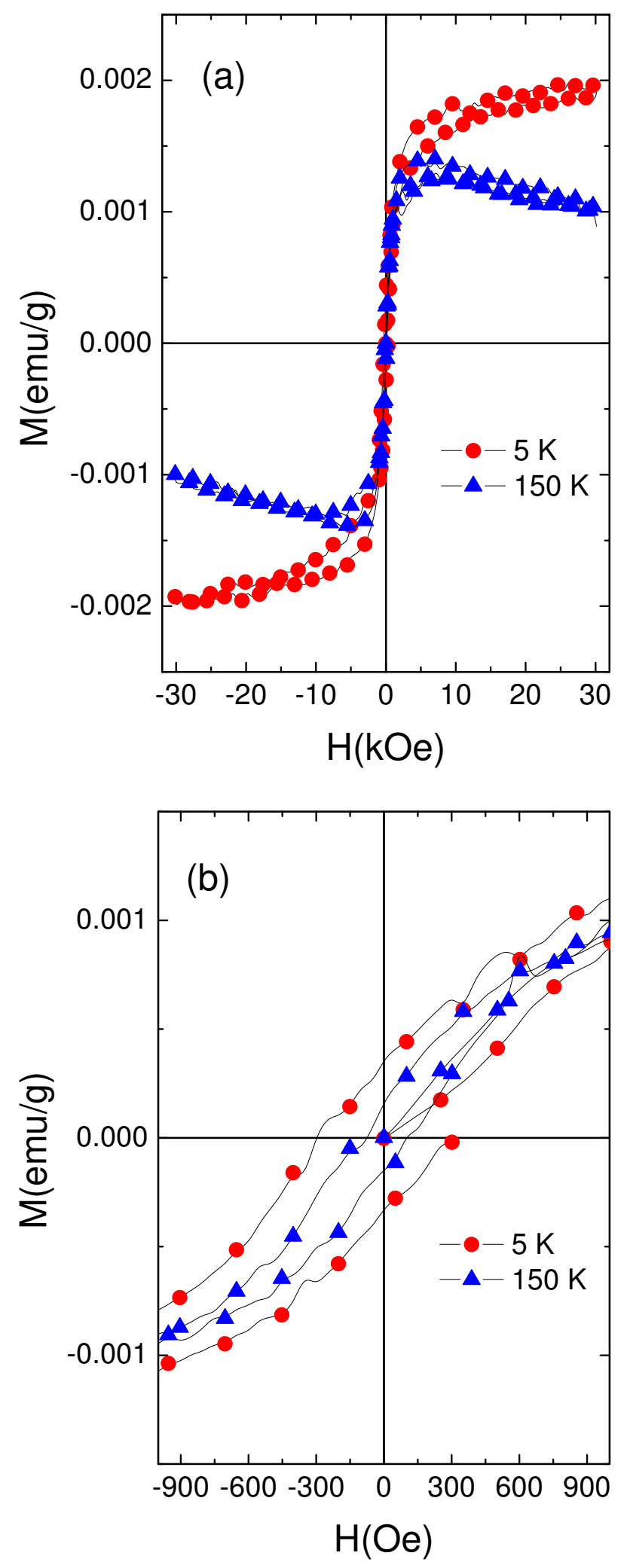

Figure 3. The hysteresis curves (taken at two temperatures, $5 K$ and $150 K$ ) for high (a) and low (b) applied magnetic field regions, showing a ferromagnetic like behavior of the NFMG sample. 
Temperature oscillations of magnetization observed in nanofluid ferromagnetic graphite6

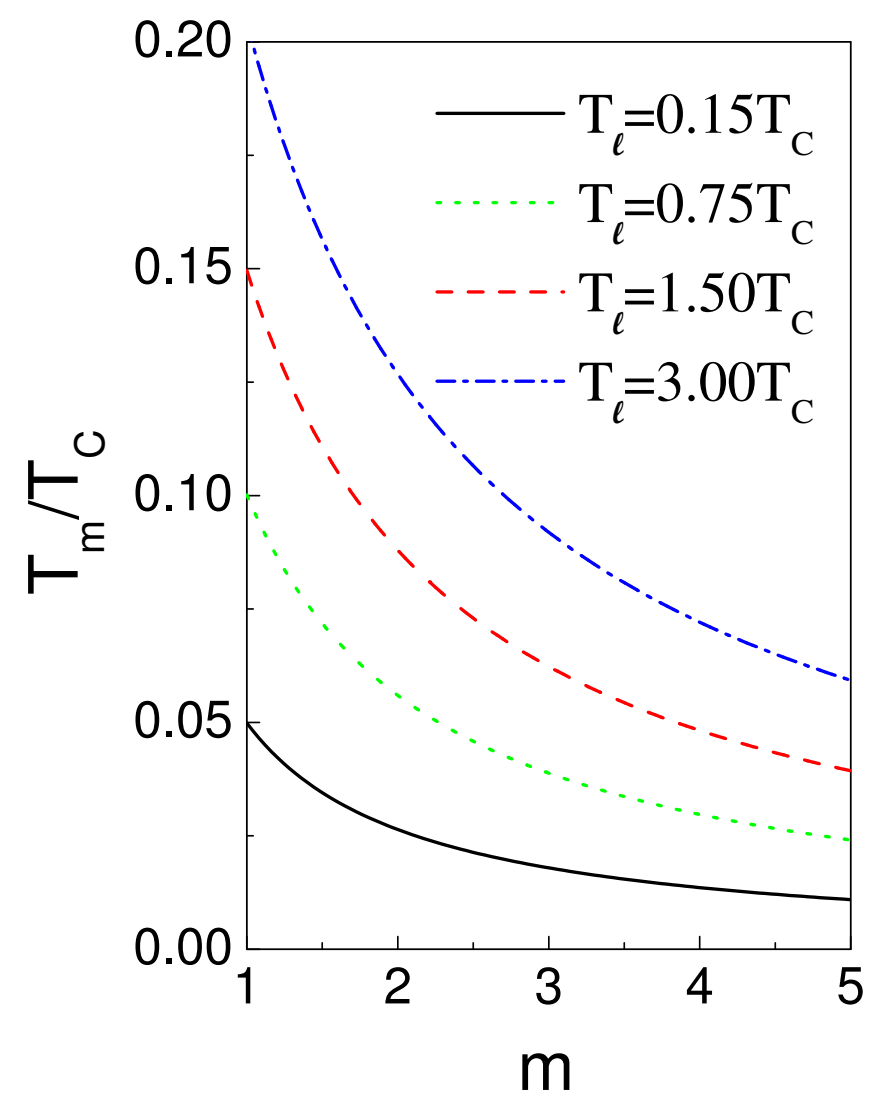

Figure 4. The predicted dependence of the reduced temperature $T_{m} / T_{C}$ on the oscillations minima $m$ for different values of the particle size $l$ related quantum temperature $T_{l}$.

de Broglie wavelength $\Lambda(T)$, in Fig, 4 we depict the solution of the above equation as the dependence of the reduced temperature $T_{m} / T_{C}$ on $m$ for different values of the particle size $l$ (in terms of the quantum temperature $T_{l} \propto \hbar^{2} / l^{2}$ ). According to this picture, the smaller the particle size (hence, the larger the quantization temperature $T_{l}$ ), the more finite size related oscillations (minima) should be observed in the temperature dependence of the magnetization $M_{l}(T)$. For example, in our particular case (with $l=10 \mathrm{~nm}$ and $\left.T_{l}=0.15 T_{C}\right)$ only first minimum $(m=1)$ is expected to be visible at non-zero temperature $T_{m}=0.05 T_{C}=15 K$, in agreement with the observations (see Fig,2).

Turning to the discussion of the high-temperature region (above $T_{p}$ ), it is quite natural to assume that the observed oscillations can be attributed to the local variation of the magnetization $\tilde{M}_{f}(r)=M_{0} g(r)$ defined by the periodic radial distribution function $r^{2} g(r)=\sin k r$ in the hard-sphere fluid model [17,18] with $k(T)=\pi M_{\infty}(T) / M_{\infty}\left(T_{p}\right) l$ where $M_{\infty}(T)$ is the above-introduced bulk magnetization of the single particle. Within this scenario, the temperature dependence of the fluid 


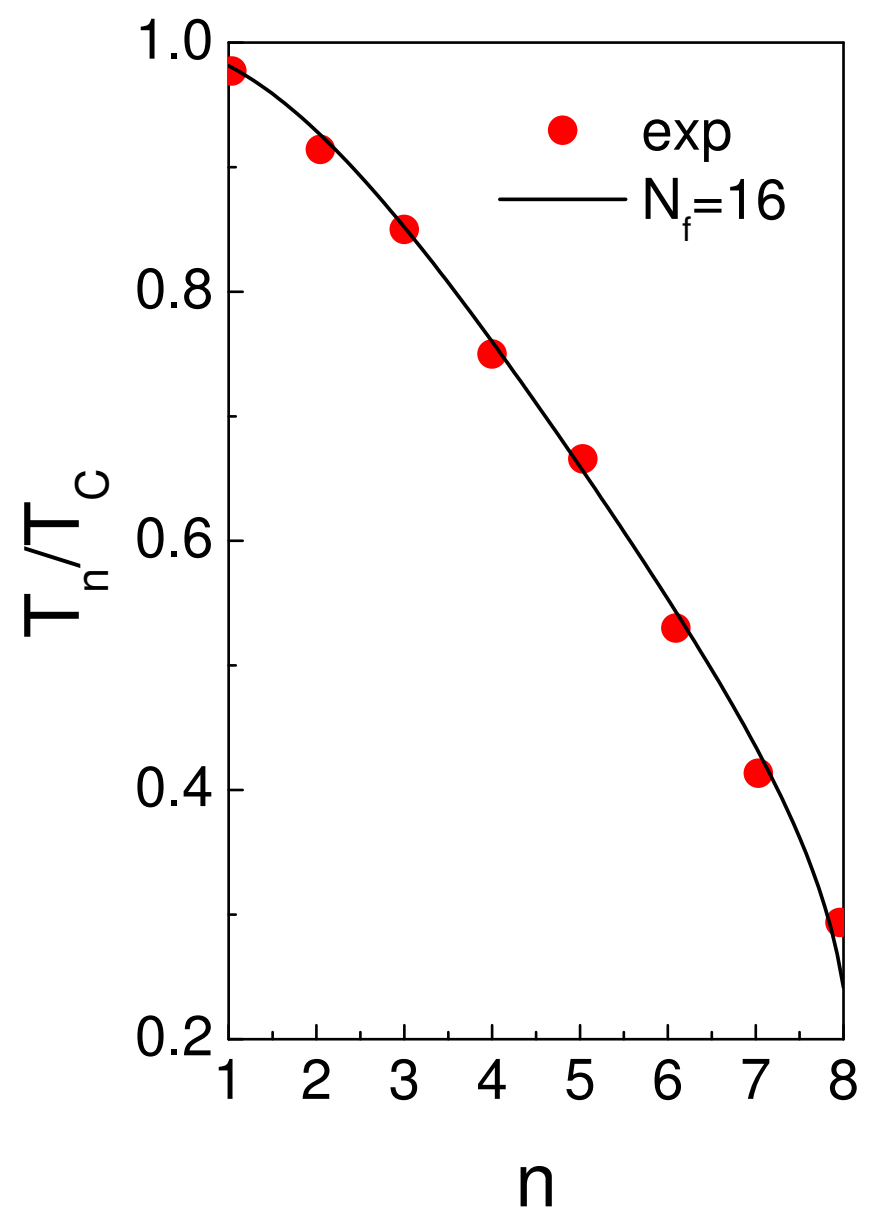

Figure 5. The predicted dependence (solid line) of the reduced temperature $T_{n} / T_{C}$ on the number of oscillations minima $n$ for $N_{f}=16$ (according to Eq.(4)) along with the extracted (from Fig, 2) experimental points.

contribution to magnetization reads:

$$
M_{f}(T)=\frac{1}{L} \int_{0}^{L} r^{2} d r \tilde{M}_{f}(r)=M_{0}\left[\frac{1-\cos k(T) L}{k(T) L}\right]
$$

where $L=N_{f} l$ and $M_{0}=\left[\pi N_{f} /\left(1-\cos \pi N_{f}\right)\right] M_{f}\left(T_{p}\right)$.

The best fits of the high-temperature data, using Eqs.(2) and (3), produced $N_{f}=L / l=16$ for the number of particles contributing to the observed oscillating behavior of nanofluid magnetization (which reasonably correlates with an average cluster size of $L=160 \mathrm{~nm}$, revealed by the TEM images of the nanofluid, see Fig.1).

Notice also that, according to Eq.(3), the number of oscillation minima $n$ of magnetization (observed at $T=T_{n}$ ) is given by the solution of the implicit equation, $k\left(T_{n}\right) L=2 \pi n$ where $n=1,2,3, \ldots$ Using the Curie-Weiss expression for bulk magnetization $M_{\infty}(T)$, the above equation results in the following explicit dependence of $T_{n}$ on $n$ and $N_{f}$ :

$$
T_{n}=\frac{T_{C}}{\sqrt{1+\left\{\tanh ^{-1}\left(2 n / N_{f}\right)\right\}^{2}}}
$$


Temperature oscillations of magnetization observed in nanofluid ferromagnetic graphite8

Fig,4 demonstrates very good agreement between the predicted $n$ dependence of $T_{n} / T_{C}$ (given by Eq.(4) with $N_{f}=16$ ) and the extracted from Fig.2 experimental points.

In summary, we reported the magnetic properties of the recently synthesized nanofluid room-temperature ferromagnetic graphite (with the single particle size of $l \simeq 10 \mathrm{~nm}$ ). In addition to a low-temperature magnetic anomaly (attributed to the manifestation of quantum size effects below $50 \mathrm{~K}$ ), we also observed strong temperature oscillations of spontaneous magnetization (attributed to manifestation of the hardsphere type pair correlations between ferromagnetic particles in the nanofluid above $50 K)$.

\section{Acknowledgments}

This work has been financially supported by the Brazilian agencies CNPq, CAPES and FAPESP.

\section{References}

[1] Miller J S and Drillon M (ed) 2002 Magnetism: Moleculales to Materials III (Weinheim: Wiley$\mathrm{VCH})$

[2] Ying Y, Gmlke E, Zhang G and Gefei W 2005 J. of Nanoscience and Nanotech. 5571

[3] Zhu H, Zhang C, Tang Y, Wang J, Ren B and Yin Y 2007 Carbon 45226

[4] Phillip J, Shima P D and Raj B 2008 Appl. Phys. Lett. 92043108

[5] Tombacz E, Bica D, Hajdu A, Illes E, Majzik A and Vekas L 2008 J. Phys.: Condens. Matter 20 204103

[6] Parkansky N, Alterkop B, Boxman R L, Leitus G, Berkh O, Barkay Z, Rosenberg Yu and Eliaz N 2008 Carbon 46215

[7] Jagodie M, Gyergyek S, Jaglicic Z, Makovec D and Trontelj Z 2008 J. Appl. Phys. 104074319

[8] Wu D, Zhu H, Wang L and Liu L 2009 Current Nanoscience 5103

[9] Widenkvist E, Boukhvalov D W, Rubino S, Akhtar S, Lu J, Quinlan R A, Katsnelson M I, Leifer K, Grennberg H and Jansson U 2009 J. Phys. D: Appl. Phys. 42112003

[10] Araújo-Moreira F M, Pardo H and Mombrú A W 2005 Patent WO/2005/123580 (Singapore: World Intellectual Property Organization)

[11] Mombrú A W, Pardo H, Faccio R, de Lima O F, Lanfredi A J C, Cardoso C A, Leite E R, Zanelatto G and Araújo-Moreira F M 2005 Phys. Rev. B 71 R100404

[12] Pardo H, Faccio R, Mombrú A W, Araújo-Moreira F M and de Lima O F 2006 Carbon 44565

[13] Faccio R, Pardo H, Denis P A, Yoshikawa Oeiras R, Araújo-Moreira F M, Veríssimo-Alves M and Mombrú A W 2008 Phys. Rev. B 77035416

[14] Souza N S, Sergeenkov S, Speglich C, Rivera V A G, Cardoso C A, Pardo H, Mombrú A W and Araújo-Moreira F M 2009 Appl. Phys. Lett. submitted

[15] Sergeenkov S, Bougrine H, Ausloos M and Cloots R 1999 JETP Lett. 69858

[16] Sergeenkov S, Mucha J, Pekala M, Drozd V and Ausloos M 2007 J. Appl. Phys. 102083916

[17] Hansen J P and Mcdonald I R 2006 The Theory of Simple Liquids (London: Academic)

[18] Scheffold F and Mason TG 2009 J. Phys.: Condens. Matter 21332102 